\title{
Computer-Assisted Distance Learning, Part I: Audiographic Teleconferencing, Interactive Satellite Broadcasts, and Technical Japanese Instruction from the University of Wisconsin-Madison
}

\author{
James L. Davis and Thomas W. Smith
}

\begin{abstract}
The primary mission of the University of Wisconsin-Madison Technical Japanese Program is to train English-speaking scientists and engineers to read technical documents written in Japanese. Semester-long courses in technical Japanese at the basic, intermediate, and advanced levels are now being offered live and interactively to students and professionals throughout the United States. Audiographic teleconferencing employs standard microcomputer hardware and a high quality sound system to share audio and visuals with many people simultaneously. Pictures, text in any writing system, data and high-resolution graphics are transmitted to participants at multiple, remote locations over ordinary telephone lines. Visuals can be annotated during the class using a graphics tablet. Alternatively, the same computer-generated image can be fed to a satellite and then broadcast throughout the country. Satellite broadcasts, coupled with the use of an audio conferencing system at each receive site, provide another effective method for interactive course delivery to sites beyond the university campus. During the three years in which technical Japanese courses have been delivered via audiographics or satellite to remote sites, student acceptance of the computer-based instructional system has been excellent. In this paper we describe the impact of audiographic teleconferencing on the teaching of technical Japanese. In a future paper we will analyze the performance of students on and off campus.
\end{abstract}

\section{The Need for Technical JaPANESE}

$\mathbf{M}$ ORE AND MORE scientists and engineers are recognizing a need for technical information that is available only in Japanese. The reasons most commonly mentioned by practicing engineers include the need to monitor technical advances by Japanese competitors, the need to read manuals and other documents received from Japanese suppliers, and the need to exchange technical information with Japanese partners in joint ventures or product development projects. The sheer volume of scientific and technical information that is generated in Japan requires that the user be selective. The rapid rate of technical progress and strict timetables for project completion require timely access to the right information. Current conditions dictate that technical professionals be able to scan

Manuscript received April 1993; revised October 1993. This work was supported in part by a grant to the University of Wisconsin-Madison from the United States Air Force Office of Scientific Research (AFOSR) as part of the United States-Japan Industry and Technology Management Training Program.

The authors are with the Department of Engineering Professional Development, University of Wisconsin-Madison, Madison, WI 53706-1573

IEEE Log Number 9400675. documents themselves, identify the information that they need immediately, and select other portions to be translated later.

Technical Japanese encompasses the vocabulary and the grammar that are used to convey scientific or technical information in Japanese. This vocabulary is almost universally ignored in the Japanese language programs that now exist at many colleges and universities. In the past scientists and engineers who wished to read technical materials written in Japanese had no recourse but to study from textbooks designed for students who were interested in Japanese history, literature, or politics. Grammatical constructions that occur primarily in technical documents were left out of conventional Japanese language curricula. Technical personnel had to learn the extensive technical vocabulary for their individual field on their own.

An additional problem facing technical professionals who wished to learn technical Japanese was the shortage of time. Conventional university programs in Japanese require three or four years, during which the student makes steady, but slow, progress. These programs perform an admirable job of producing well-rounded individuals who can read, write, comprehend, and converse in Japanese. However, the extremely limited number of American scientists and engineers who can function in Japanese, even at a rudimentary level, is testimony to the failure of conventional Japanese language programs to serve the needs of technical professionals in the United States.

\section{TECHNICAL JAPANESE EDUCATION AT THE UNIVERSITY OF WISCONSIN-MADISON}

The goal of the Technical Japanese Program from its inception has been to help scientists and engineers obtain from Japanese books, technical articles and other documents the important information that they need to carry out their own work more effectively. With this goal in mind two textbooks [1], [2] designed specifically to teach technical Japanese to English speakers have been coauthored by two faculty members from the UW-Madison and a colleague in Japan: Edward E. Daub (now Emeritus Professor, Engineering Professional Development), R. Byron Bird (now Emeritus Professor, Chemical Engineering), and the late Nobuo Inoue (formerly Professor, Mechanical Engineering, Science University of Tokyo). Reading remains the primary focus of the program. However, 
activities are being introduced to develop students' listening comprehension and conversational skills. The following options are currently available to students and professionals:

\section{A. Basic Technical Japanese (Three Courses, One Calendar Year)}

This option is recommended for advanced students (seniors or graduate students) and professionals who have never studied Japanese, but need to read technical Japanese books and documents in their own fields. Students who require a strong foundation in reading and need only limited exposure to conversation will benefit from these courses. The first two courses introduce the three types of Japanese script (hiragana, katakana, and 365 frequent kanji) and most of the grammar necessary for reading in the sciences and engineering. The final course includes information on resources for monitoring Japanese scientific and technical information, and concludes with individual projects whereby participants explore recent Japanese journal articles or patents in their own fields.

\section{B. Summer Translation Seminar (Eight Weeks)}

An intensive summer program of directed study is available for intermediate or advanced students of Japanese or technical Japanese who wish to focus on technical translation in their own field of interest. Each participant selects his or her own documents for translation. Japanese technical articles, equipment manuals, and patent disclosures are the types of documents most frequently chosen. Class meetings consist of problem solving sessions, with each student learning from the problems encountered by the other students.

\section{Certificate in Japanese Studies for Engineering Majors (Seven Courses, Minimum One Calendar Year)}

This option is recommended for undergraduate students who seek a balance among comprehension of technical Japanese, the ability to converse in Japanese, and an understanding of Japanese culture. The required courses comprise two semesters of introductory Japanese language and two semesters of intermediate level technical Japanese. In the former courses, students learn the foundations of reading, writing, listening, and speaking. In the latter courses, students reinforce their foundation in grammar, learn $500 \mathrm{kanji}$ that appear frequently in technical articles, build a technical vocabulary, and gain extensive practice in translating technical passages. The remaining three courses may be selected by the student from offerings in Japanese art, history, literature, or politics. Undergraduate students normally take one of the seven courses each semester in addition to their required engineering courses. Most students graduate in four years with a degree in engineering and the Japanese studies certificate, although some students go on to take additional courses and graduate with a B.A. in Japanese in addition to a B.S. in engineering. Certificate programs in Technical Japanese Studies for graduate students and professionals are also available.

\section{UW/Japan Engineering Leadership Program (Five Years)}

Undergraduates accepted into this program study engineering and Japanese at the UW-Madison for three and one half years, then spend one year carrying out a senior research project at a Japanese university. Some students obtain internships at companies in Japan during the summer vacation of this year. Students then return to Madison for one semester to complete the requirements for their bachelor's degree. Corporate sponsorship of this program permits each student to receive financial aid, the majority of which is distributed during the year in which the student lives in Japan. In addition to their regular engineering courses, students in this program study the Japanese language for four semesters, study intermediate level technical Japanese for two semesters, and take one additional course in Japanese history or politics.

\section{E. Advanced Technical Japanese (One Semester)}

Undergraduates, graduate students, and professionals who have completed one year of technical Japanese focus on current articles from Japanese scientific and technical journals in rapidly changing fields. Sample topics include IC design/manufacture, advanced materials, artificial intelligence, telecommunications, and robotics. New topics and readings are selected each year.

\section{Characteristics OF Audiographics}

"Audiographics" is an accepted term in the fields of telecommunications and distance education. It denotes the combination of audio conferencing with still frame graphics. The Department of Engineering Professional Development (EPD) at the UW-Madison has been using audiographics for course delivery since 1968. New audiographics technology recently adopted by EPD operates on standard microcomputers connected by high-speed modems over ordinary phone lines. The current system is far more efficient and flexible than its predecessors and offers more complex display and communications options.

This audiographics system combines multi-point audio conferencing with computer-based display of graphics, animation and full color pictures. The system is fully interactive: A student at any site can talk with the instructor or with students at any other site, and can ask a question or use a graphics tablet to present text or a drawing on the computer screen at any time. All sites share the audio and visual information equally. The high resolution ( 1,280 line) computer display provides full color and approximately four times the resolution of standard television. This resolution is sufficient to read a page such as this comfortably on the screen. Since the system displays still images, rather than moving pictures, some think of it as inferior to television. This is not the case. The interactive capability, high resolution display, ease of use, and ability to reach any location where there is a phone line, make modern audiographics comparable in many ways even to interactive television, and it can be used at a fraction of the cost.

In addition to technical Japanese, EPD offers a variety of other courses to engineers and technical managers via audiographics. These courses are presently focused in man- 
ufacturing, are generally noncredit courses, 12-30 hours in length, and are delivered over a number of sessions - usually twice a week for one to two hours. The system has also been used with excellent results for semester-long graduate seminars in electrical engineering and quality product design, and for a foundation course in reading German.

Audiographics is ideally suited for international course sharing. EPD has used audiographics to deliver courses to Mexico and to Europe, and is cooperating with European universities on course development and delivery. Another goal is trans-Pacific conferencing, which would allow Asian students and faculty to participate in University of Wisconsin courses.

\section{TeChNiCAL JAPANESE AND Distance Education}

The audience for technical Japanese is both demanding and widely disbursed. That is, the number of people in any one location who may need this skill is relatively small, yet there are many locations where the skill is needed. (Over $75 \%$ of the engineers who have participated in technical Japanese courses to date have been either electrical engineers or employed in the electronics industry.) Face-to-face instruction on site is not possible, the short course format is not effective, and an extended stay at a major university center, such as Madison, requires an unacceptably long absence from work. The Japanese language instruction available in most locations does not address the special needs of the technical professional, as described above. This problem is compounded by the shortage of programs in technical Japanese. Only a handful of universities in the U.S. currently offer programs on this topic. Distance education is therefore a practical necessity.

All courses in both the basic and intermediate technical Japanese sequences, as well as the advanced course, carry graduate credit from the University of Wisconsin-Madison and also from the National Technological University (NTU). NTU, an accredited private university engaged in distance education for engineers at the post-graduate level, provides courses from 45 member universities to engineers and managers at 459 locations throughout the country using a satellite broadcast network [3]. The electronics industry is heavily represented among the 132 organizations that sponsor NTU courses for their employees.

The two options of audiographic teleconferencing and interactive satellite broadcasts make it possible to deliver technical Japanese courses to professionals anywhere in the United States. The students at a specific site choose the technology that is more convenient for delivery of the desired course to their site. For sites using audiographics all visuals are prepared in advance on a microcomputer, and are preshipped to remote sites over telephone lines or on diskettes. Japanese and English sentences are created using a front-end processor and a Japanese word processing program. Japanese text is input using kana (Japanese phonetic symbols). The front-end processor then permits selective conversion from kana into kanji (ideographic characters). Each screen of information is saved as a separate file. Each file becomes a separate slide in the audiographic information delivery system, as described above. Because of the intricate structure of Japanese characters, the text is prepared using special fonts and large type, and high-resolution monitors are employed. Students who receive the visuals by satellite need only tune the receiver to the correct location and frequency. The use of an audio conferencing system at each site, whether an audiographics site or a satellite site, is essential in order for real time, instructor-student interaction.

Simultaneous audio and video communication during the class provides an excellent learning environment for students at remote sites. The utilization of audiographic teleconferencing or interactive satellite broadcasts allows engineers in government or corporations to participate in technical Japanese classes without leaving their office or conference room. In the basic sequence classes meet for 50 minutes per day, four days a week, during the regular fifteen-week semesters. Normally, class time is divided between lectures by the instructor on aspects of Japanese grammar and translations by the students of selected Japanese passages. The instructor illustrates grammatical points with example sentences in Japanese, which are displayed simultaneously on monitors at all sites. Additional features of the software, such as annotations in different colors, highlighting, and a zoom function, provide the instructor with all of the tools available in a traditional classroom setting. Students use their microphones to ask questions of the instructor, and the instructor responds. Discussion between the instructor and the students, or among students at different sites, is a regular feature of every class session. Special "question and answer" sessions are also scheduled outside regular class meetings, to take the place of the "office hours" that are available to students on campus. In these Q\&A sessions students at remote sites have the opportunity to ask more detailed questions than they could ask during the regular class period. Providing many opportunities for communication between the off-campus student and the instructor has proven to be essential to maintain student motivation when dealing with difficult material over the long academic year.

In addition to regular participation in class sessions, students on and off campus submit daily assignments. Students off campus return their completed exercises to the instructor via fax or electronic mail. During class exercises of various types are displayed on the monitors. Students have the opportunity to ask questions about each exercise as it is discussed in class. Each audiographics site is equipped with a graphics tablet and pen, identical to those used by the instructor. Thus, students at different sites can present alternative ideas or interpretations visually in the "shared space" of the computer screen during class. An optical scanner, which may be employed at each site, can be used to introduce supplemental material at the last minute or during class. This feature makes the medium of audiographic teleconferencing ideal for any course that involves extensive problem solving or teamwork.

\section{Course CONDUCT and Evaluation}

The use of audiographic teleconferencing or interactive satellite broadcasts offers many advantages in terms of access to a large pool of students who may be distributed among many 
TABLE I

ENROLLMENT IN BASIC TECHNICAL JAPANESE I

\begin{tabular}{|c|c|c|}
\hline \multicolumn{3}{|c|}{ 1990-1991 } \\
\hline Remote & 1 site & 7 students \\
\hline UW-Madison & 1 site & 9 students \\
\hline Total & 2 sites & 16 students \\
\hline \multicolumn{3}{|c|}{ 1991-1992 } \\
\hline Remote & 5 sites & 22 students \\
\hline UW-Madison & 1 site & 11 sludents \\
\hline Total & 6 sites & 33 sludents \\
\hline \multicolumn{3}{|c|}{$1992-1993$} \\
\hline Remote & 18 cites & 38 students \\
\hline UW-Madison & 1 cite & 12 sludents \\
\hline Total & 19 cites & 50 students \\
\hline
\end{tabular}

sites, interactive instruction, the potential for students to make steady progress over a moderate period of time, and the relatively low cost of course delivery. However, several features of instruction require additional preparation and attention on the part of the instructor. In the traditional classroom the very presence of the instructor provides a measure of control over the students. The instructor can see every student, can hear every comment, and can judge immediately the reaction of the students to the course material as the class proceeds. In the technical Japanese classes full-time and part-time students are always present at the instructor's site, so immediate feedback from a portion of the class is possible. However, since the majority of the students in each class are now located at remote sites (e.g., see Table I), mechanisms must be developed to obtain regular feedback from these students.

Although all participants have equal access to the information displayed on the monitors, students who receive the courses by audiographics cannot see the instructor nor can the instructor see them. Students who receive the courses by interactive satellite broadcast can see the instructor, but no one else can see those students. When microphones at one site are turned on, everyone can hear comments made by students at that particular site, but when the microphones at a particular site are turned off, no one at any other site can hear the comments or discussion among participants at that site. Thus, maintaining participant attention and interest is a critical consideration in course planning. In particular, avoiding lengthy lectures, stopping frequently to invite questions, and providing ample opportunity for discussion by participants are extremely important. Varying the class format and building into each class period some activity that requires student participation are effective ways of maintaining participant interest and attention. Providing sufficient opportunities for questions reduces the risk that participants at one site will still be discussing a previous point "off mike" even though the rest of the class has moved on to a new topic.

The basic technical Japanese sequence was offered via audiographics to one corporate site in Wisconsin from September, 1990 to August, 1991, and to five corporate sites in the Midwest during the 1991-92 academic year. During the 1992-93 academic year students at one government site participated via audiographics and students at 17 corporate and government sites around the United States participated by means of interactive satellite broadcasts. Each semester stu-
TABLE II

STUDENT EVALUATION OF DELIVERY METHOD

\begin{tabular}{c|c|c}
\hline Year & Campus & Remote \\
\hline \multicolumn{3}{c}{ was compatabile with my learning stye. } \\
\hline $1990-91$ & 4.8 & 5.0 \\
$1991-92$ & 6.7 & 6.2 \\
$1992-93$ & 6.3 & 6.0 \\
\hline Statement 2: The delivery system used for this course is an effective \\
\multicolumn{3}{c}{ medium for this type of subject matter. } \\
\hline $1990-91$ & 4.3 & 5.0 \\
$1991-92$ & 7.0 & 6.4 \\
$1992-93$ & 6.6 & 6.1 \\
\hline Statement 3: I could learn as effectively using this delivery system as I \\
could in a traditional (face-to-face) classroom. \\
\hline $1990-91$ & 3.3 & 4.9 \\
$1991-92$ & 7.0 & 5.9 \\
$1992-93$ & 6.4 & 5.4 \\
\hline Statement 4: I would take another course delivered in this way. \\
\hline 1990-91 & 4.5 & 6.1 \\
$1991-92$ & 7.0 & 6.2 \\
$1992-93$ & 6.0 & 6.2 \\
\hline Statement 5: It would have been difficult for me to get the information \\
presented in this program in any other way. \\
\hline 1990-91 & 4.1 & 5.0 \\
$1991-92$ & 5.3 & 6.2 \\
1992-93 & 3.5 \\
\hline
\end{tabular}

dents were also present at the instructor's site in Madison. The enrollment figures for these three years appear in Table I. Tables II-V contain a portion of the results of the course evaluation carried out each year. ( In Tables II-IV, the rating scale is as follows: $7=$ strongly agree, $4=$ neutral, and $1=$ strongly disagree.) This evaluation was designed specifically for courses that employ audiographic teleconferencing, but is also applicable for interactive satellite broadcasts. Because of the small class sizes, a rigorous statistical analysis was not meaningful, but a few conclusions can be drawn by inspecting the results contained in these tables. In general, participants at all sites rated the computer-based instruction and delivery system highly. The trend toward more positive student evaluation over time can be attributed in part to greater experience by the instructor and support staff at the University of Wisconsin-Madison in working with the system and in part to conscious efforts to improve the system based on the results of these very evaluations.

In 1990, the first year that the current computer-based system was used, participants at the remote site were briefed in advance regarding the type of technology that would be used in the course, and had an opportunity to practice using the microphone and tablet. No such briefing was given to students on campus. The results of the evaluation given to the 1990-91 group of students indicate that the level of satisfaction on the part of the campus students was consistently lower than that of the remote students. Some students at the instructor's site were initially resistant to the idea of using a microphone to address an instructor in the same room, but the benefits of the system and the advantages of participation by students from both the academic and corporate environment fostered acceptance by all students within a short period of time. In subsequent years some form of orientation for all participants, both on and off campus, regarding the system was carried out 
TABLE III

STUDENT EVALUATION OF VISUAL SYSTEM FEATURES

\begin{tabular}{|c|c|c|}
\hline Year & Campus & Remote \\
\hline \multicolumn{3}{|c|}{ Statement 6: I could see the visuals on the video monitor without difficulty. } \\
\hline $1990-91$ & 5.2 & 6.3 \\
\hline $1991-92$ & 6.3 & 6.7 \\
\hline $1992-93$ & 6.5 & 5.4 \\
\hline \multicolumn{3}{|c|}{$\begin{array}{l}\text { Statement 7: The transmission time needd to transmit a visual in its entirety } \\
\text { did not detract from the instruction. }\end{array}$} \\
\hline $1990-91$ & 4.3 & 5.6 \\
\hline $1991-92$ & 5.7 & 6.0 \\
\hline $1992-93$ & 6.5 & 6.1 \\
\hline \multicolumn{3}{|c|}{$\begin{array}{l}\text { Statement } 8: \text { The instructor's use of the tablet to annotate the visuals and } \\
\text { write down discussion points helped my understanding of the content. }\end{array}$} \\
\hline $1990-91$ & 4.8 & 5.7 \\
\hline $1991-92$ & 6.3 & 6.6 \\
\hline $1992-93$ & 6.6 & 6.5 \\
\hline \multicolumn{3}{|c|}{ Statement 9: Annotations were clear and readable. } \\
\hline $1990-91$ & 4.4 & 5.7 \\
\hline $1991-92$ & 6.7 & 6.2 \\
\hline $1992-93$ & 6.6 & 6.5 \\
\hline
\end{tabular}

TABLE IV

STUDENT EVALUATION OF TECHNICAL SYSTEM

\begin{tabular}{c|c|c}
\hline Year & Campus & Remote \\
\hline \multicolumn{3}{c}{$\begin{array}{c}\text { Statement 10: The instructor's voice was clear and distinct as it came over } \\
\text { the audio teleconferencing system. }\end{array}$} \\
\hline $1990-91$ & 6.3 & 5.3 \\
$1991-92$ & 6.7 & 6.9 \\
$1992-93$ & 6.6 & 6.5 \\
\hline Statement 11: The voices of the students at other teleconferencing locations \\
were clear and distinct as they came over the audio teleconferencing \\
\multicolumn{3}{|c}{ system. } \\
\hline 1990-91 & 3.8 \\
$1991-92$ & 6.3 & 2.9 \\
$1992-93$ & 5.9 & 4.2 \\
\multicolumn{3}{|c}{ interfere with my learning of the content. } \\
\hline Statement 12: Any distruptions in the audio or visual systems appeared to \\
be minor to me. \\
$1990-91$ & 4.3 & 4.3 \\
$1991-92$ & 5.3 & 5.2 \\
$1992-93$ & 6.1 & 5.2 \\
\hline \multicolumn{3}{|c|}{3.9} \\
\hline
\end{tabular}

prior to actually starting the course. The results from students on campus improved from a marginally positive response in 1990-91 to strongly positive responses in both 1991-92 and 1992-93 (Statements 1-4).

Students at the remote sites were much more open to the use of this kind of technology, since without this medium they would not have been able to participate in these courses at all (Statement 5). The quality of visuals at each site depends in part on the facilities used for viewing the images transmitted by audiographic or broadcast by satellite. Following the first evaluation the course in Madison was moved from a long, narrow room that was used for several different functions to a square room that became the dedicated audiographics classroom for the College of Engineering. Thus, students were able to sit closer to the monitors and could see the instructor and the monitors at the same time. The specific facilities
TABLE V

GENERAL INFORMATION

\begin{tabular}{c|c|c}
\hline Year & Campus $(\%)$ & Remote $(\%)$ \\
\hline Statement 14: I have taken a course by teleconferencing before. \\
\hline $1990-91$ & 33 & 44 \\
$1991-92$ & 10 & 50 \\
$1992-93$ & \multicolumn{2}{|c}{ Campus/Remote: $6 \%$} \\
\hline \multicolumn{3}{|c}{ Catement 15: I would like to take a course using this delivery system } \\
\hline \multicolumn{3}{|c}{ again. } \\
\hline Campus/Remote: $88 \%$ \\
$1990-91$ & 100 & 89 \\
$1992-93$ & 86 & 100 \\
\hline
\end{tabular}

available and the room layout employed at remote sites are major variables that influence the responses to Statement 6 .

Preshipping slides to remote sites before class reduced the transmission time required to actually present visuals during class (Statement 7). Partitioning of the computer memory to increase efficiency also contributed to reduced transmission time. Annotations by the instructor were valued highly by students both on and off campus (Statement 8). Experience in using the system allowed the instructor to gradually make better use of the drawing features (colored pens, highlighting, outlining in different colors, etc.) available in the audiographic teleconferencing software (Statement 9).

Students consistently reported hearing the instructor clearly, and students on campus were able to hear the students off campus, but students off campus continued to experience difficulty in hearing the students on campus (Statements 10 and 11). This disparity is attributed to some resistance on the part of campus students to using the microphones, coupled with less experience on the part of campus students in speaking "in public." Students off campus were, in general, more concerned with disruptions in the audio or visual systems than were their campus counterparts (Statements 12 and 13). This is quite reasonable, since any disruption that might occur would leave the off-campus students temporarily unable to participate in the class session.

Statement 14 indicates the disparity in experience with teleconferencing between students on and off campus. Despite the range of previous experience, roughly $90 \%$ or more of the students each year expressed interest in taking another course using the technology employed here (Statement 15). It is recognized that the target audience for these courses, engineers and engineering students, represents a group that would be expected to accept technology in the classroom more readily than would the population at large. Classes were generally conducted from Madison, but during the first two years for one class session each semester the instructor visited a corporate site and conducted the class from that site. Meeting the participants at a "remote" site early in the semester, and soliciting comments from participants both on and off campus allowed the instructor and technical support staff to make changes in course format early each semester to address some of the issues presented by the students. As the number of remote sites increased, meeting all of the offcampus participants in person became impractical, but efforts to communicate with off-campus students by telephone, fax, and especially electronic mail were increased. 


\section{SUMMARY}

Understanding technical Japanese is a skill that is increasingly in demand as corporations focus more attention on Japan and Japanese technology. In distance learning modern technology provides the instructor with all of the tools available in a conventional classroom. Audiographic teleconferencing is an ideal medium for delivering technical Japanese, or any other course, live and interactively to a national or international audience. The basic sequence in technical Japanese has been successfully delivered live via audiographic teleconferencing and by interactive satellite broadcasts to a remote audience throughout the United States. Acceptance of the technology by students has been excellent. Important considerations when teaching to a remote audience via audiographics have been identified, and improvements in the system have been made. In a subsequent paper the examination performance of students at the remote sites will be compared with the performance of full-time students at the UW-Madison.

\section{REFERENCES}

[1] E. E. Daub, R. B. Bird, and N. Inoue, Comprehending Technical Japanese. Madison, WI: University of Wisconsin Press. 1975.

[2] E. E. Daub, R. B. Bird, and N. Inoue, Basic Technical Japanese. Madison, WI: University of Wisconsin Press, 1990.

[3] National Technological University, Annual Report 1991-1992. Fort Collins, CO: National Technological University, 1992.

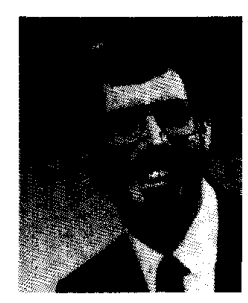

James L. Davis earned his B.S. in chemical engineering from the University of Rochester and his M.S. and Ph.D. in Forestry (Wood Chemistry) from the University of Wisconsin-Madison.

Currently, he is Assistant Professor of Technical Japanese at the University of Wisconsin-Madison. A licensed Professional Engineer in New York and Wisconsin, he has worked as a chemical engineer in industry and conducted research as a Fulbright Graduate Research Fellow at Kyoto University in Kyoto, Japan. In addition to research publications and conference presentations in the U. S. and in Japan, he has nine years of experience as a translator of Japanese technical materials. He currently teaches basic, intermediate, and advanced courses in technical Japanese and has presented short courses and written articles on monitoring Japanese technical information. He also writes "Words and Kotoba," a quarterly column in the Newsletter of the Japanese Language Division of the American Translators Association.

Thomas W. Smith earned his A.B. at Dartmouth College and M.S. at the University of Wisconsin-Madison.

$\mathrm{He}$ is Director of Engineering Telecommunications Programming at the University of Wisconsin-Madison. Before joining the University of Wisconsin. he worked in city and state government as well as private industry. His responsibilities in telecommunications include strategic planning and facilities development, as well as day-to-day management and budgeting of courses delivered via satellite, audiographics, videotape, and computer conferencing. $\mathrm{Mr}$. Smith has written a number of papers and articles on telecommunications and distance education, and is a frequent speaker on this topic. He also directs short courses in Distance Education. 